\title{
Influence of Oxygen on Growth of Carbon Thin Films
}

\author{
Prabhat Kumar $^{1}$, Mukul Gupta ${ }^{1, a)}$, D. M. Phase ${ }^{1}$ and Jochen Stahn ${ }^{2}$ \\ ${ }^{1} U G C-D A E$ Consortium for Scientific Research, University Campus, Khandwa Road, Indore-452001, India \\ ${ }^{2}$ Laboratory for Neutron Scattering and Imaging, Paul ScherrerInstitut, CH-5232 Villigen PSI, Switzerland \\ a)mgupta@csr.res.in
}

\begin{abstract}
In this work we studied the influence of oxygen gas on growth of carbon thin films in a magnetron sputtering process. X-ray absorption spectroscopy (XAS), x-ray and neutron reflectivity techniques were used to probe carbon thin films deposited with and without oxygen at room temperature. XAS in particularly x-ray absorption near edge spectroscopy (XANES) is powerful technique to identify the nature of hybridization of carbon atoms with other elements. In a XANES pattern, presence of $\mathrm{C}=\mathrm{O}$ and $\mathrm{C}-\mathrm{O}$ bonds is generally observed in spite of the fact that oxygen has not been deliberately included in the growth process. In order to confirm the presence of such features, we introduced a small amount of oxygen at $1 \%$ during the growth of carbon thin films. Though such additions do not affect the number density as observed by x-ray and neutron reflectivity, they severally affect the C K-edge spectra as evidenced by an enhancement in carbon-oxygen hybridization. Observed results are helpful in analyzing the C K-edge spectra more confidently.
\end{abstract}

\section{INTRODUCTION}

Amorphous carbon $(a-C)$ is well-known for its excellent hardness and chemically inertness [1-4]. It is widely used as a protective coating for magnetic recording media [5-7], biomedical devices. $a$-C thin films can be deposited by any non-equilibrium processes e.g. pulse laser deposition (PLD), magnetron sputtering (MS), etc. Depending on the technique used and deposition condition, its hardness can be varied. One of the frequently used term for the quality of hardness measurement of the film is $\mathrm{sp}^{3} / \mathrm{sp}^{2}$ hybridization ratio.

In this context $\mathrm{C} \mathrm{K}$-edge XANES is one the most widely used technique for determination of electronic structure of materials. It can probe the local structure around an element [8]. It's sensitivity to any small changes in the local structure of the material is remarkable.C K-edge XANES pattern has been explored by several researchers and some ambiguity can be seen among tiny features present there. In C K-edge XANES a feature appearing at $285.5 \mathrm{eV}$ is due to $\mathrm{sp}^{2}$ hybridization of carbon and it is generally dominated in its graphitic form [9-10]. Another feature arising at $292.6 \mathrm{eV}$ is assigned as $\mathrm{sp}^{3}$ hybridization of carbon [11-13] and it is dominated in diamond. On the other hand, in $a$-C both these features can be seen but their relative intensities vary. In addition, tiny features appearing around photo energies of 2867.6 and $288.8 \mathrm{eV}$ have also been observed in several works. Several attempts have been made to understand the origin of such features. In a recent work by Ray et. al [14], these features have been explained due to hybridization of carbon with oxygen $(\mathrm{C}=\mathrm{O}$ and $\mathrm{C}-\mathrm{O})$.In an another work by Benny et. al [15],C K-edge XANES spectra of carbon nanotubes oxidized in the presence of $\mathrm{O}_{3} /$ wet-air and shown that these features are due to hybridization of carbon with oxygen. However, presence of $\mathrm{C}=\mathrm{O}$ and $\mathrm{C}-\mathrm{O}$ features, even as a tiny kink in carbon thin films deposited by PLD and MS is rather surprising as oxygen has not been deliberately incorporated during the growth. In order to clarify the origin of these feature, we have deliberately used a tiny flow of oxygen during sputtering of a carbon target with Argas. Observed C K-edge XANES of samples prepared with and without oxygen gas are discussed and they indeed help to identify features present in the XANES pattern unambiguously. 


\section{EXPERIMENTAL DETAILS}

Thin films of carbon were deposited using direct current magnetron sputtering (dcMS) at room temperature using Orion-8, AJA Int. Inc. thin film deposition system. The base pressure of the chamber was of the order of $1 \times 10^{-}$ ${ }^{7}$ Torrand working pressure was kept at 3 mTorr. A 3-inch carbon target of purity $99.99 \%$ and argon gas of purity $(99.9995 \%)$ are used for deposition. We deposited two set of carbon thin films on Si substrate. First using pure argon and another with a mixture of $1 \%$ oxygen gas of purity (99.95\%) - gas flow used for Ar and oxygen were 20 and $0.2 \mathrm{sccm}$. Both samples were deposited at $100 \mathrm{~W}$ sputtering power for 2 hours and thickness achieved were about $50 \mathrm{~nm}$. During deposition, substrate was continuously rotated at $60 \mathrm{rpm}$ for better uniformity and target to substrate distance was kept fixed at $12 \mathrm{~cm}$.Resulting films were characterized for their thickness using x-ray reflectivity (XRR) by a standard diffractometer (Bruker D8 Discover) equipped with $\mathrm{Cu} \mathrm{k}_{\alpha} \mathrm{X}$-ray source. Since the scattering contrast between $\mathrm{Si}$ and $\mathrm{C}$ is poor for x-rays, we also did neutron reflectivity (NR) measurements. For neutrons the scattering length density of $\mathrm{Si}$ and $\mathrm{C}$ are 4.1 and $6.65 \mathrm{fm}$, respectively. Clearly since the contrast between film and substrate is larger, it is expected that precise information about number density can be measured precisely with NR. NR measurements have been carried out in the time of flight mode using Selene setup at AMOR at SINQ/PSI, Switzerland [16,17]. The electronic structure of the samples was determined using XANES technique at the C K-edge in total electron yield (TEY) mode at BL01 beamline at Indus-2 synchrotron radiation source RRCAT Indore. To remove surface contamination, sample were sputter cleaned in situ using an Ar ions incident at an angle of $45^{\circ}$.

\section{RESULTS AND DISCUSSION}

XRR and NR pattern of as-deposited samples are shown in fig. 1 and fig. 2, respectively. In both cases thickness oscillations can be seen clearly but they are more pronounced in NR due to larger scattering contrast. Detailed fitting of both was done using Parratt 32 software [18] and obtained fitting parameters are shown in table1. Both from XRR and NR measurements we find that the thickness of C films prepared with $0 \%(1 \%) \mathrm{O} 2$ are about $515(450) \AA$ and are similar within experimental errors. Roughness of films are also similar. Though XRR measurements show that the number densities are similar for 0 and $1 \% \mathrm{O} 2$ samples, they are clearly less for $1 \% \mathrm{O}_{2}$ sample from NR measurements. However we also find that $\operatorname{Im} \rho$ increases significantly with addition of oxygen (see table 1). Since oxygen has a smaller SLD than $\mathrm{C}$ at $5.8 \mathrm{fm}$, it is expected that the addition of $\mathrm{O}$ in $\mathrm{C}$ would result in overall smaller SLD. Also deposition rate changes from $4.3 \AA / \mathrm{min}$ to $3.8 \AA / \mathrm{min}$ when $\mathrm{O}_{2}$ gas flow increases from 0 to $1 \%$.

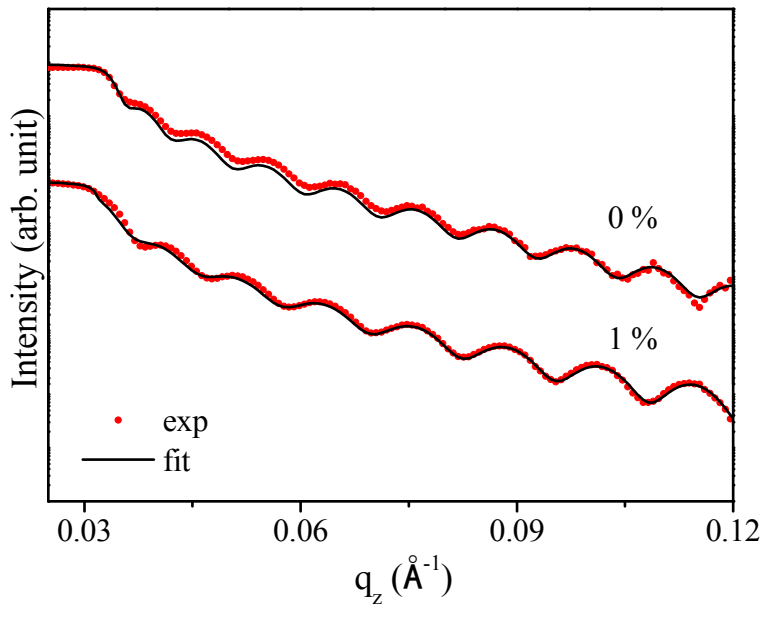

Fig.1.XRR pattern of $\mathrm{C}$ thin films with 0 and $1 \% \mathrm{O}_{2}$ gas flow.

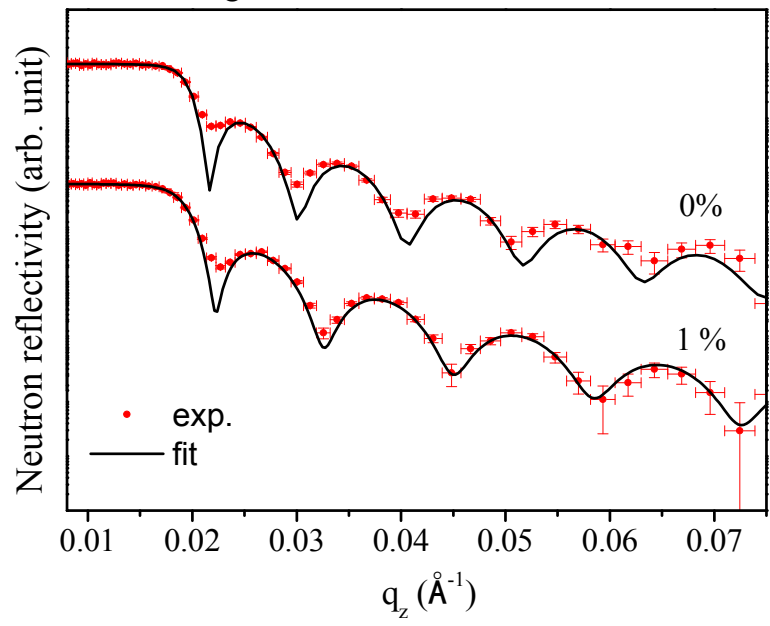

Fig.2.NR pattern of $\mathrm{C}$ thin films with 0 and $1 \% \mathrm{O}_{2}$ gas flow.

Though from XRR measurements the influence of addition of $1 \%$ oxygen gas can only be seen in terms of variations in deposition rates, NR measurements clear yield information about the change in $\rho$. It will be interesting to see how the local environment gets affected with addition of oxygen. We did XANES measurement of asdeposited samples at $\mathrm{C}$ and $\mathrm{O}$ k-edges and background removed spectra (using Athena software [19])are shown infigure. 3 and 4, respectively.At carbon k-edge (fig. 3), we see features labeled as $a, b, c$ and $d$, the feature $a(285.5$ 
$\mathrm{eV}$ ) is due to $1 \mathrm{~s} \rightarrow \pi^{*}$ transition. It can be clearly seen that feature a is intense in $0 \%$, whereas it gets suppressed in 1 $\%$, however $\mathrm{c}$ and $\mathrm{d}$ enhance with addition of $\mathrm{O}_{2}$. Clearly, they arise due to hybridization of between carbon and oxygen. Here feature $c$ and $d$ arises due to $\mathrm{C}=\mathrm{O}$ and $\mathrm{C}-\mathrm{O}$ following literature whereas the nature of feature $b$ remains unclear at the moment. It may be noted that since XANES in TEY mode is a surface sensitive technique, it is expected that the surface may get contaminated when samples are exposed to environment. To remove such contaminations samples were sputter cleaned with an Ar ion source working at $5 \mathrm{keV}, 50 \mu \mathrm{A}$ and incident at an angle of about $45^{\circ}$.

Table. 1: Fitted parameters for $\mathrm{C}$ thin films prepared using 0 and $1 \% \mathrm{O}_{2}$ obtained from XRR and NR measurements.

\begin{tabular}{|l|l|l|l|l|}
\hline \multicolumn{5}{|c|}{ X-ray Reflectivity } \\
\hline $\mathrm{O}_{2} \%$ & Thickness $(\AA, \pm 5 \AA)$ & $\rho\left(\AA^{-2}\right)$ & $\operatorname{Im} \rho\left(\AA^{-2}\right)$ & Roughness $(\AA, \pm 2 \AA)$ \\
\hline 0 & 514 & $2.2(5) \times 10^{-5}$ & $8.5 \times 10^{-7}$ & 12 \\
\hline 1 & 450 & $2.1(5) \times 10^{-5}$ & $2.0 \times 10^{-6}$ & 12 \\
\hline \multicolumn{5}{|c|}{ Neutron Reflectivity } \\
\hline 0 & 518 & $6.4(5) \times 10^{-6}$ & 0 & 12 \\
\hline 1 & 447 & $6.1(5) \times 10^{-6}$ & 0 & 11 \\
\hline
\end{tabular}

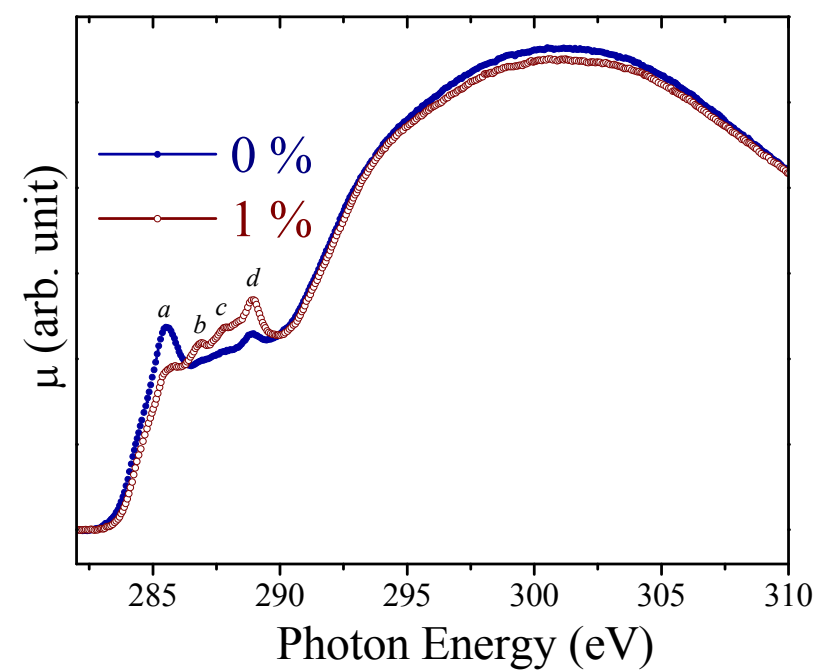

Fig.3.XAS at carbon k-edge of as-deposited with various $\mathrm{N}_{2}$ partial pressure.

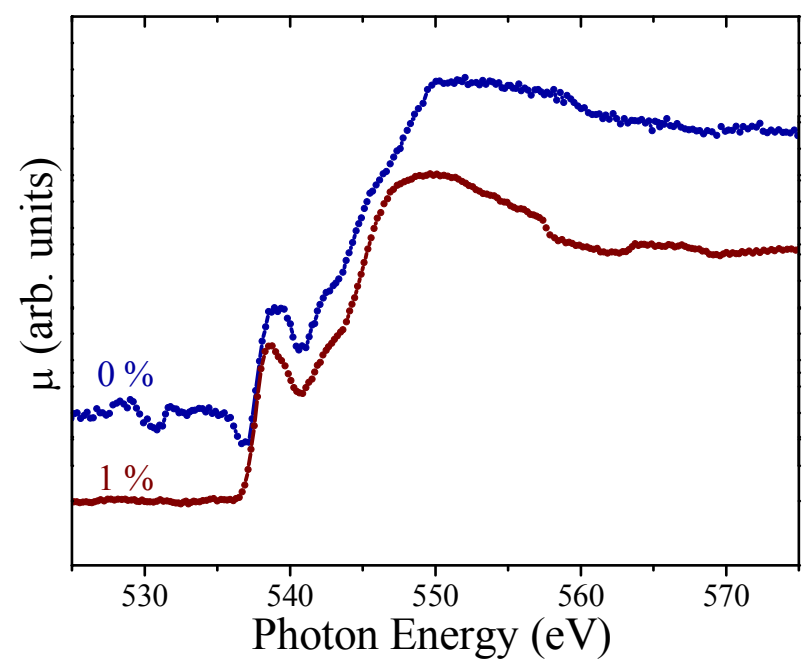

Fig.4.XAS at nitrogen k-edge of as-deposited with various $\mathrm{N}_{2}$ partial pressure.

In case of oxygen k-edge, we see only a weak signature for $0 \%$ and it gets enhanced with addition of $\mathrm{O}_{2}$ of $1 \%$ of total gas flow. Therefore, our XANES measurement clearly, reveal the presence of $\mathrm{O}_{2}$ and rise in feature $\mathrm{c}$ and $\mathrm{d}$ becomes unambiguous and has origin of carbon and oxygen hybridization.From the oxygen k-edge, we can see that in pure carbon film oxygen is almost in background and further incorporation of the oxygen edge is dominating, this signifies the presence of oxygen in the film.

\section{CONCLUSION}

From this study, we have investigated the influence of $\mathrm{O}_{2}$ on the local structure of carbon. It appears that even a small amount of carbon present during growth of carbon thin film influences the carbon k-edge XANES spectra and faint features present there have been clearly resolved by us. The presence of the $\mathrm{O}_{2}$ has the visible impact on deposition rate as well as on the number density probed by neutron reflectivity. 


\section{ACKNOWLEDGMENTS}

Author is thankful to Mr. LayantaBehera, Mr. Anil Gome in sample preparation and XRR measurement. Mr.

Rakesh Sah and Mr. R. Bhimgade for their help in XANES measurements.We are thankful to Dr. A. K. Sinha and Dr. V. Ganesan for support and encouragement.

\section{REFERENCES}

1. J. Robertson, Mater. Sci. Eng. R 37, 129 (2002).

2. A. Erdemir and C. Donnet, J. Phys. D 39, R311 (2006).

3. J. Robertson, Phys. Status Solidi A 205, 2233 (2008).

4. J. Robertson, Jpn. J. Appl. Phys. 50, 01AF01 (2011).

5. J.Windeln, C.Bram, H.-L. Eckes, D. Hammel, J. Huth, J.Marien, et al., Appl.Surf.Sci.179 (2001) 167.

6. $\quad$ E.C. Cutiongco, D. Li, Y.W. Chung, C.S. Bathia, IEEE Trans. Magn. 33 (1997) 938.

7. A. Leson, H.Hilgers, Phys.Blatter 55 (1999) 63.

8. J. E. PENNER-HAHN, The University of Michigan, Ann Arbor, MI, USA, 01063.

9. P. Skytt, P. Glans, D. C. Mancini, J.-H. Guo, N. Wassdahl, and J. Nordgren, Phy. Rev. B, 50, 457-461(1994).

10. A. J. Maxwell, P. A. Brühwiler, D. Arvanitis, J. Hasselström, and N. Mårtensson, Phys. Rev. B, 79, 15671570(1997).

11. Y. Ma, N. Wassdahl, P. Skytt, J. Guo, J. Nordgren, P. D. Johnson, J-E. Rubensson, T. Boske, W. Eberhardt, and S. D. Kevan, Phys. Rev. Lett., 69, 2598-2601(1992).

12. I. Ahmad, S.S. Roy, M.A. Rahman, T.I.T. Okpalugo, P.D. Maguire, J.A. McLaughlin, Curr. Appl. Phys. 9, 937-942(2009).

13. J. Stöhr, NEXAFS Spectroscopy, Springer Series in Surface Sciences, vol. 25Springer, Berlin, 1991.

14. Sekhar C. Ray, W.F. Pong, P. Papakonstantinou, , Thin Solid Films 610,42-47(2016).

15. TirandaiHemraj-Benny, Sarbajit Banerjee, SharadhaSambasivan, MahalingamBalasubramanian, Daniel A. Fischer, GyulaEres, Alexander A. Puretzky, David B. Geohegan, Douglas H. Lowndes, Weiqiang Han, James A. Misewich, and Stanislaus S. Wong, Small, 2, 26-35(2006).

16. M. Gupta, T. Gutberlet, J. Stahn, P. Keller, D. Clemens, Pramana J. Phys. 63, 57 (2004).

17. J. Stahn, A. Glavic, Nuclear Instruments and Methods A 821, 44-54 (2016).

18. L.G. Parratt, Phys. Rev. B. 95, 359-369(1954).

19. B. Ravel and M. Newville, Journal of Synchrotron Radiation 12, 537-541 (2005). 